\title{
Fronts from two-dimensional dispersal kernels: Beyond the nonoverlapping-generations model
}

\author{
Daniel R. Amor and Joaquim Fort \\ Departament de Física, Universitat de Girona, Girona, 17071 Catalonia, Spain \\ (Received 30 July 2009; revised manuscript received 21 October 2009; published 23 November 2009)
}

\begin{abstract}
Most integrodifference models of biological invasions are based on the nonoverlapping-generations approximation. However, the effect of multiple reproduction events (overlapping generations) on the front speed can be very important (especially for species with a long life spam). Only in one-dimensional space has this approximation been relaxed previously, although almost all biological invasions take place in two dimensions. Here we present a model that takes into account the overlapping generations effect (or, more generally, the stage structure of the population), and we analyze the main differences with the corresponding nonoverlappinggenerations results.
\end{abstract}

DOI: 10.1103/PhysRevE.80.051918

PACS number(s): 87.23.Cc, 89.20.-a, 89.75.Fb

\section{INTRODUCTION}

Reaction-diffusion and reaction-dispersal fronts have many applications in physical, biological, and crossdisciplinary systems [1-3], e.g., virus infection fronts [4,5], combustion fronts $[6,7]$, human population fronts $[8,9]$, etc. Motivated by Reid's paradox of rapid tree range expansions, recently we have proposed a framework which is useful in two-dimensional (2D) space under the assumption of nonoverlapping generations [10]. Modeling forest postglacial recolonization fronts by using single-kernel reaction-dispersal assumptions results in the underestimates of the observed speeds (this disagreement is known as Reid's paradox). In order to better predict such speeds, our recent work introduced several-component kernels (with characteristic distances differing several orders of magnitude) [10]. In this way, long-distance dispersal (even if occurring infrequently) makes it possible to predict speeds of the right order of magnitude, as observed from postglacial tree recolonization fronts.

However, previous work in two dimensions did not take the age structure of tree populations into account. Indeed, trees reproduce every year and not only once in their lifetime, so generations clearly overlap. Therefore, here we will extend the 2D model [10] to overlapping generations. We shall show that the corrections (relative to the nonoverlapping approximation) are relevant, which justifies the importance of our model. Previously, overlapping-generation models have been only developed in one dimension [11-18]. Our model is relevant not only to tree species, but can be applied to compute front speeds also in other biophysical and physical systems in which the reproductive (or reactive) process happens more than once for each individual (or particle).

\section{EVOLUTION EQUATION}

\section{A. Nonstructured populations in two dimensions (continuous space random walk)}

Integrodifference equations have been widely used to model biophysical and cross-disciplinary reaction-dispersion phenomena. For example, for the case of trees population dispersion (seed dispersal) takes place just after reproduction (seed production). Thus the evolution of a nonstructured population in a $2 \mathrm{D}$ space is driven by the well-known integrodifference equation [19]

$$
\begin{aligned}
p(x, y, t+T)= & R_{0} \int_{-\infty}^{+\infty} \int_{-\infty}^{+\infty} p\left(x+\Delta_{x}, y+\Delta_{y}, t\right) \\
& \times \phi\left(\Delta_{x}, \Delta_{y}\right) d \Delta_{x} d \Delta_{y},
\end{aligned}
$$

where $p(x, y, t+T)$ is the population density at the location $(x, y)$ and time $t+T$. Recently we have argued that this evolution equation is also relevant to other biological species besides trees, e.g., humans [20]. However, for the sake of definiteness and clarity, in this paper we will consider trees in our explanations. The time interval $T$ is that between two subsequent dispersal events or "jumps" (in the nonstructured model, $T$ is one generation, i.e., the mean age of trees when they begin to produce seeds). $R_{0}$ is the net reproductive rate (number of seeds per parent tree and year which survive into an adult tree). Equation (1) is the nonoverlappinggenerations model. It is worth to stress that in this model, the net reproductive rate per year is always used for $R_{0}$ [19]. The dispersal kernel $\phi\left(\Delta_{x}, \Delta_{y}\right)$ is the probability per unit area that a particle that a seed falling from a parent tree located at $\left(x+\Delta_{x}, y+\Delta_{y}, t\right)$ reaches the ground at $(x, y, t+T)$. Strictly, Eq. (1) is valid only at sufficiently low values of the population density $p$, because there is a maximum saturation density above which net reproduction vanishes (see Eq. (9) in Ref. [20]); however, this point does not affect the computation of front speeds because, as we shall see below, such computations can be performed at low values of $p$.

Equation (1) is a continuous space random-walk (CSRW) equation in two dimensions. It is just an integration over all possible jumps, which takes into account the probability of each possible jump [dispersal kernel $\phi\left(\Delta_{x}, \Delta_{y}\right)$ ] as well as the productivity of new individuals (net reproduction rate $R_{0}$ ).

Let us first summarize some previous results, and we will then extend them to more general situations. The speed of fronts evolving according to Eq. (1) can be obtained under some general assumptions, as follows [10,21]. We assume that $R_{0}>1$, that the initial population density has bounded support [i.e., that $p(x, y, t)$ vanishes outside a finite region], and that for $t \rightarrow \infty$ the front becomes approximately planar at scales much larger than that of individual dispersal events. 
Then we can choose the $x$ axis parallel to the local velocity of the front (i.e., $c \equiv\left|c_{x}\right|$ and $c_{y}=0$ ). Finally, we look for constant-shape solutions with the form $p=p_{0} \exp [-\lambda$ $(x-c t)]$ at $x-c t \rightarrow \infty$ (leading edge of the invasion front). Requiring that $\lambda>0$ yields the asymptotic $(t \rightarrow \infty)$ front speed for 2D nonstructured populations [10,21],

$$
c=\min _{\lambda>0} \frac{\ln \left[R_{0} \hat{\varphi}(\lambda)\right]}{\lambda T},
$$

where

$$
\hat{\varphi}(\lambda) \equiv \int_{0}^{\infty} d \boldsymbol{\Delta} \varphi(\boldsymbol{\Delta}) I_{0}(\lambda \boldsymbol{\Delta}),
$$

and

$$
I_{0}(\lambda \boldsymbol{\Delta}) \equiv \frac{1}{2 \pi} \int_{0}^{2 \pi} d \theta \exp [\lambda \boldsymbol{\Delta} \cos \theta]
$$

is the modified Bessel function of the first kind and order zero. The dispersal probability per unit area $\phi(\Delta)$ is related to that per unit length $\varphi(\Delta)$ (i.e., into a 2D ring of area $2 \pi \boldsymbol{\Delta} d \boldsymbol{\Delta})$ according to [10]

$$
\varphi(\Delta)=2 \pi \Delta \phi(\Delta) .
$$

We have also assumed an isotropic dispersion kernel (i.e., that $\phi$ depends only on distance $\boldsymbol{\Delta} \equiv \sqrt{\Delta_{x}^{2}+\Delta_{y}^{2}}$ ), and applied the normalization of probability, i.e.,

$$
\int_{0}^{\infty} d \boldsymbol{\Delta} \varphi(\boldsymbol{\Delta})=1
$$

\section{B. Structured populations in two dimensions}

In the previous section we have summarized the nonstructured (or nonoverlapping) model in two dimensions [10,21]. It is widely used because of its mathematical simplicity (let us mention that it has been often formulated in one dimension [19,21], because then it becomes even simpler). But obviously, assuming that individuals reproduce only once in their lifetime is not a good approximation in general. Indeed, trees produce new seeds during many years. Thus we should expect intuitively that multiple reproduction events will lead to faster values of the front speed (and, in some cases, even prevent the extinction of the population). For these reasons, we have built a model that makes it possible to predict front speeds for structured populations spreading across 2D space.

\section{Introductory example}

Let us use a simple example to introduce the main assumptions and equations of our 2D overlapping model. Consider a species with a life span of three years. If one-year-old individuals cannot produce seeds, in the nonstructured model $T=2$ yr. Thus, the limitation of the nonstructured model (1) is that it does not take into account the reproduction of threeyear-old individuals in this simple example.

Let us assume that reproduction events take place only once per year (this is valid for most tree species, as seed production happens only during some period in fall). In order to compute the production of new individuals, we have to take into account the contribution of both the two-year-old and the three-year-old subpopulations. Therefore,

$$
\begin{aligned}
p_{1}(x, y, t+1)= & R_{02} \int \phi_{2}\left(\boldsymbol{\Delta}_{x}, \boldsymbol{\Delta}_{y}\right) p_{2}\left(x+\boldsymbol{\Delta}_{x}, y+\boldsymbol{\Delta}_{y}, t\right) d \boldsymbol{\Delta}_{x} d \boldsymbol{\Delta}_{y} \\
& +R_{03} \int \phi_{3}\left(\boldsymbol{\Delta}_{x}, \boldsymbol{\Delta}_{y}\right) p_{3}\left(x+\boldsymbol{\Delta}_{x}, y+\boldsymbol{\Delta}_{y}, t\right) d \boldsymbol{\Delta}_{x} d \boldsymbol{\Delta}_{y},
\end{aligned}
$$

where $p_{1}(x, y, t+1)$ is the population density of one-year-old individuals at location $(x, y)$ and time $t+1$ (time is measured in years). The right-hand-side of Eq. (7) contains two terms. The first one is the contribution of two-year-old individuals. One can easily observe that this term is analogous to Eq. (1): $R_{02}$ is the net reproductive rate of two-year-old individuals, $\phi_{2}\left(\boldsymbol{\Delta}_{x}, \boldsymbol{\Delta}_{y}\right)$ is the dispersal kernel, and $p_{2}\left(x+\boldsymbol{\Delta}_{x}, y+\boldsymbol{\Delta}_{y}, t\right)$ is the two-year-old population density at location $\left(x+\boldsymbol{\Delta}_{x}, y\right.$ $+\boldsymbol{\Delta}_{y}$ ) and time $t$. Similarly, the last term describes the reproduction and dispersal of seeds produced by three-year-old individuals. In order to predict the invasion speed, we need to know the population densities of adult individuals in future times. These population densities are governed by the following equations:

$$
\begin{aligned}
& p_{2}(x, y, t+1)=p_{1}(x, y, t), \\
& p_{3}(x, y, t+1)=p_{2}(x, y, t),
\end{aligned}
$$

because in this model, for simplicity, we neglect the effect of deaths in subpopulations 1 and 2 (i.e., all one-year-old individuals will be two years old after one year has elapsed, etc.). Finally we have the following set of equations that describes our structured population:

$$
\begin{gathered}
p_{1}(x, y, t+1)=\int R_{0} \phi\left(\boldsymbol{\Delta}_{x}, \boldsymbol{\Delta}_{y}\right) p_{2}\left(x+\boldsymbol{\Delta}_{x}, y+\boldsymbol{\Delta}_{y}, t\right) d \boldsymbol{\Delta}_{x} d \boldsymbol{\Delta}_{y} \\
+\int R_{0} \phi\left(\boldsymbol{\Delta}_{x}, \boldsymbol{\Delta}_{y}\right) p_{3}\left(x+\boldsymbol{\Delta}_{x}, y+\boldsymbol{\Delta}_{y}, t\right) d \boldsymbol{\Delta}_{x} d \boldsymbol{\Delta}_{y} \\
p_{2}(x, y, t+1)=p_{1}(x, y, t), \\
p_{3}(x, y, t+1)=p_{2}(x, y, t) .
\end{gathered}
$$

For simplicity, here we have assumed that the net reproductive rate and the dispersal kernel of two- and three-yearold individuals are the same. Similarly to the nonstructured model, we look for constant-shape solutions, but now for each subpopulation, i.e., $p_{1}(x, y, t)=w_{1} \exp [-\lambda(x-c t)]$, $p_{2}(x, y, t)=w_{2} \exp [-\lambda(x-c t)], \quad$ and $\quad p_{3}(x, y, t)=w_{3} \exp$ $[-\lambda(x-c t)]$. Then we can rewrite Eq. (10) as

$$
\begin{aligned}
w_{1} \exp (\lambda c)= & R_{0} w_{2} \int_{0}^{\infty} \varphi(\boldsymbol{\Delta}) I_{0}(\lambda \boldsymbol{\Delta}) \boldsymbol{\Delta} d \boldsymbol{\Delta} \\
& +R_{0} w_{3} \int_{0}^{\infty} \varphi(\boldsymbol{\Delta}) I_{0}(\lambda \boldsymbol{\Delta}) \boldsymbol{\Delta} d \boldsymbol{\Delta},
\end{aligned}
$$




$$
\begin{aligned}
& w_{2} \exp (\lambda c)=w_{1}, \\
& w_{3} \exp (\lambda c)=w_{2},
\end{aligned}
$$

where $I_{0}(\lambda \boldsymbol{\Delta})$ and $\varphi(\boldsymbol{\Delta})$ are defined by Eqs. (4) and (5). If $w_{1}, w_{2}$, and $w_{3}$ are thought of as the entries of a array

$$
\vec{w}=\left(\begin{array}{l}
w_{1} \\
w_{2} \\
w_{3}
\end{array}\right)
$$

then the system (11) can be rewritten as

$$
\exp (\lambda c) \vec{w} \equiv \vec{H}(\lambda) \vec{w},
$$

where $\vec{H}(\lambda)$ is the matrix

$$
\vec{H}(\lambda)=\left(\begin{array}{ccc}
0 & R_{0} \int_{0}^{\infty} \varphi(\Delta) I_{0}(\lambda \boldsymbol{\Delta}) \boldsymbol{\Delta} d \boldsymbol{\Delta} & R_{0} \int_{0}^{\infty} \varphi(\boldsymbol{\Delta}) I_{0}(\lambda \boldsymbol{\Delta}) \boldsymbol{\Delta} d \boldsymbol{\Delta} \\
1 & 0 & 0 \\
0 & 1 & 0
\end{array}\right) .
$$

It is well known that the front speed $c$ for systems with the form (12) can be obtained from [11]

$$
c=\min _{\lambda>0} \frac{\ln \left[\rho_{1}(\lambda)\right]}{\lambda},
$$

where $\rho_{1}$ is the largest of the eigenvalues of $\vec{H}(\lambda)$.

Equation (14) is the result for the front speed of structured populations in two dimensions. Some results exist in one dimension [11], but in that case the Bessel function $I_{0}(\lambda \boldsymbol{\Delta})$ does not appear at all and the solutions are rather different. Indeed, in order to compare the one- and two-dimensional models, below we present the main one-dimensional (1D) equations and we assign some hypothetical values to the parameters.

In one dimension, clearly the kernel per unit area $\phi\left(\Delta_{x}, \Delta_{y}\right)$ has no physical meaning. The $1 \mathrm{D}$ evolution equation [equivalent to Eq. (1)] is obviously

$$
p(x, t+T)=R_{0} \int_{-\infty}^{+\infty} p\left(x+\Delta_{x}, t\right) \widetilde{\varphi}\left(\Delta_{x}\right) d \Delta_{x},
$$

where we have not used the notation $\varphi(\Delta)$ for the kernel in order to avoid confusion with the 2D model. Let us assume a very simple dispersal kernel so that seeds can be only dispersed at a specific distance $\boldsymbol{\Delta}_{0}$ (with probability $\frac{1}{2}\left(1-p_{e}\right)$ along each direction of propagation) or not dispersed at all (with probability $p_{e}$, which is called the persistence),

$$
\begin{aligned}
\widetilde{\varphi}\left(\Delta_{x}\right)= & p_{e} \delta\left(\boldsymbol{\Delta}_{x}\right)+\frac{1}{2}\left(1-p_{e}\right) \delta\left(\boldsymbol{\Delta}_{x}-\boldsymbol{\Delta}_{0}\right) \\
& +\frac{1}{2}\left(1-p_{e}\right) \delta\left(\boldsymbol{\Delta}_{x}+\boldsymbol{\Delta}_{0}\right),
\end{aligned}
$$

where $\delta\left(\boldsymbol{\Delta}_{x}\right)$ is the 1D Dirac delta. It is easy to extend Eqs. (15) and (16) to the overlapping case by performing the same steps as in the 2D example above (i.e., assuming a threestage population, looking for constant-shape solutions $p_{i}(x, y, t)=w_{i} \exp [-\lambda(x-c t)]$, etc. $)$. This yields

$$
h_{i j}^{1 \mathrm{D}}= \begin{cases}R_{0}\left[\begin{array}{c}
p_{e}+\frac{1}{2}\left(1-p_{e}\right) \exp \left(-\lambda \Delta_{0}\right) \\
+\frac{1}{2}\left(1-p_{e}\right) \exp \left(\lambda \Delta_{0}\right)
\end{array}\right], & \text { if } i=1, j=2,3 \\
1, & \text { if } i=2, j=1 \\
1, & \text { if } i=3, j=2 \\
0, & \text { elsewhere, }\end{cases}
$$

where $h_{i j}^{1 \mathrm{D}}$ are the elements of the matrix $\overline{\overline{H_{1 \mathrm{D}}}}(\lambda)$. Such a matrix is the analog to Eq. (13), but now describing onedimensional spread [12-18]. Instead of the 2D Eq. (14), we obtain for the speed of the front in one dimension

$$
c_{1 \mathrm{D}}=\min _{\lambda>0} \frac{\ln \left[\rho_{1 \mathrm{D}}(\lambda)\right]}{\lambda},
$$

where $\rho_{1 \mathrm{D}}$ is the largest of the eigenvalues of $\overline{\overline{H_{1 \mathrm{D}}}}(\lambda)$.

In order to compare to this $1 \mathrm{D}$ invasion speed, in two dimensions we also consider a kernel corresponding to isotropic jumps at a single distance $\boldsymbol{\Delta}=\boldsymbol{\Delta}_{0}$,

$$
\varphi(\Delta)=p_{e} \delta(\Delta)+\left(1-p_{e}\right) \delta\left(\Delta-\Delta_{0}\right),
$$

and the dispersal kernel (19) must be introduced in $\vec{H}(\lambda)$ [see Eq. (13)] in order to solve the 2D invasion speed.

In order to present some estimates of the $1 \mathrm{D}$ and $2 \mathrm{D}$ speeds, let us assign some reasonable numerical values to the parameters [10]. We assume that the typical jump distance of our hypothetical species is $\boldsymbol{\Delta}_{0}=100 \mathrm{~m}$. We take the value of 0.98 for the probability $p_{e}$ (i.e., the probability that a seed is dispersed $100 \mathrm{~m}$ away from its parent tree is $2 \%$ ). Then, Table I shows 1D and 2D front speeds [obtained by solving Eq. (18) and (14), respectively] for several values of $R_{0}$. 
TABLE I. Front speeds in one and two dimensions (in $\mathrm{m} / \mathrm{yr}$ ) and the error of the $1 \mathrm{D}$ speed (relative to the 2D speed $c$ ) for an example with $n=3$ stages and several values of the net fecundity $R_{0}$ [measured in seeds/(tree* yr)].

\begin{tabular}{rccc}
\hline \hline$R_{0}$ & $c_{1 \mathrm{D}}$ & $c$ & $\begin{array}{c}\text { Error } \\
(\%)\end{array}$ \\
\hline 4 & 30.8 & 24.4 & 20.7 \\
10 & 39.6 & 32.5 & 17.9 \\
40 & 48.4 & 40.7 & 15.9 \\
\hline \hline
\end{tabular}

It is seen from Table I that the $1 \mathrm{D}$ model leads to an overestimation of the $2 \mathrm{D}$ speeds, and the overestimation is more important for low values of $R_{0}$. This justifies the need to tackle the 2D case for nonoverlapping generations, as first done in the present paper. Still, Eq. (14) holds only for populations with a life span of 3 years. Below we tackle the general case of an arbitrary number of years $n$, and apply it to an example.

\section{Spread of structured populations in two dimensions}

In this section we generalize the introductory example (presented above) to deal with a population structured into $n$ subpopulations. Although we apply the method to tree species, our model can be extended to other invasive species (like animals). Then one should take into account some specific features (e.g., the fact that for trees dispersal is simultaneous with reproduction) and make new assumptions (e.g., for birds dispersal is more frequent in young individuals), but we plan to tackle such cases in future work. The main point here is to note that building the reaction-dispersal matrix $\vec{H}(\lambda)$ is an important step in order to solve the front speed problem. In the following lines we explain how to obtain the matrix $\stackrel{\vec{H}}{(}(\lambda)$ by using two simpler matrices. At the end of the section we will use the reaction-dispersal matrix to find a new general equation for the invasion speed of structured populations in two dimensions.

In order to introduce the notation, let us first return to our simple example (three-stage system). If dispersal were not present, obviously

$$
\begin{gathered}
p_{1}(x, y, t+1)=R_{0} p_{2}(x, y, t)+R_{0} p_{3}(x, y, t), \\
p_{2}(x, y, t+1)=p_{1}(x, y, t), \\
p_{3}(x, y, t+1)=p_{2}(x, y, t),
\end{gathered}
$$

and Eq. (20) would provide the population dynamics of the three stages, governed only by reproduction. Using a simpler notation,

$$
\vec{p}(x, y, t+1)=\vec{A} \vec{p}(x, y, t),
$$

Where

$$
\vec{p}(x, y, t)=\left(\begin{array}{l}
p_{1}(x, y, t) \\
p_{2}(x, y, t) \\
p_{3}(x, y, t)
\end{array}\right)
$$

From Eq. (20),

$$
\vec{A}=\left(\begin{array}{ccc}
0 & R_{0} & R_{0} \\
1 & 0 & 0 \\
0 & 1 & 0
\end{array}\right) \text {. }
$$

$\vec{A}$ can be called the demographic matrix. Element $a_{i j}$ of this matrix represents the rate at which an individual in state $j$ gives rise to individuals in state $i$ per unit time (in this case one year). For example, element $a_{12}=R_{0}$ is the productivity of new (one-year old) individuals from a two-year old individual. $a_{32}=1$ means that all two-year-old individuals will be three years old a year later (and similarly for $a_{21}=1$ ). The zeros of the matrix represent the transitions that are forbidden under our assumptions. For example, $a_{23}=0$ because a three-year old individual will never become neither produce a two-year-old individual a year later.

Let us now add stage-specific dispersal into this description. Similarly to $1 \mathrm{D}$ studies $[11,23]$, we introduce $\phi_{i j}\left(\boldsymbol{\Delta}_{x}, \boldsymbol{\Delta}_{y}\right)$ as the probability that an individual making the transition from stage $i$ to stage $j$ moves from location $\left(x+\boldsymbol{\Delta}_{x}, y+\boldsymbol{\Delta}_{y}\right)$ to location $(x, y)$. If there is no dispersal during a given transition, the associated kernel is the 2D Dirac delta function.

Instead of Eq. (20), taking into account dispersal events we obtain the set

$$
\begin{aligned}
p_{i}(x, y, t+1)= & \int_{-\infty}^{+\infty} \sum_{j=1}^{3} \phi_{i j}\left(\boldsymbol{\Delta}_{x}, \boldsymbol{\Delta}_{y}\right) a_{i j} p_{j} \\
& \times\left(x+\boldsymbol{\Delta}_{x}, y+\boldsymbol{\Delta}_{y}, t\right) d \boldsymbol{\Delta}_{x} d \boldsymbol{\Delta}_{y},
\end{aligned}
$$

with $i=1,2,3$. Again, the notation can be simplified by creating a dispersal matrix $\vec{\Phi}$. In our example,

$$
\vec{\Phi}=\left(\begin{array}{ccc}
\delta_{2 \mathrm{D}}(\boldsymbol{\Delta}) & \phi\left(\boldsymbol{\Delta}_{x}, \boldsymbol{\Delta}_{y}\right) & \phi\left(\boldsymbol{\Delta}_{x}, \boldsymbol{\Delta}_{y}\right) \\
\delta_{2 \mathrm{D}}(\boldsymbol{\Delta}) & \delta_{2 \mathrm{D}}(\boldsymbol{\Delta}) & \delta_{2 \mathrm{D}}(\boldsymbol{\Delta}) \\
\delta_{2 \mathrm{D}}(\boldsymbol{\Delta}) & \delta_{2 \mathrm{D}}(\boldsymbol{\Delta}) & \delta_{2 \mathrm{D}}(\boldsymbol{\Delta})
\end{array}\right) .
$$

We can now rewrite Eq. (23) in vector form,

$$
\vec{p}(x, y, t+1)=\int_{-\infty}^{+\infty} \overrightarrow{\vec{\Phi}} \circ \vec{A} \vec{p}\left(x+\boldsymbol{\Delta}_{x}, y+\boldsymbol{\Delta}_{y}, t\right) d \boldsymbol{\Delta}_{x} d \boldsymbol{\Delta}_{y},
$$

where the symbol $\circ$ stands for the Hadamard product [22], i.e., $(\overrightarrow{\vec{\Phi}} \circ \vec{A})_{i j}=\phi_{i j} a_{i j}$. It is very important to note that Eq. (25) remains valid if we extend our study to a population with a larger number of stages (say, $n$ stages). In this case, the matrices $\vec{\Phi}$ and $\vec{A}$ are both square matrices of order $n$. This is the general case we will now deal with. 
For each subpopulation, we look for constant-shape solutions (e.g., $p_{i}(x, y, t)=w_{i} \exp [-\lambda(x-c t)]$, as in the example above). Whereas in the three-stage population (considered above) using this into Eq. (25) leads to Eq. (11), in the general case of $n$ stages we obtain

$$
\exp (\lambda c) \vec{w}=\left(\int_{-\infty}^{+\infty} \vec{\Phi} \circ \vec{A} \exp [-\lambda \Delta \cos \theta] d \Delta d \theta\right) \vec{w} .
$$

Assuming an isotropic dispersal kernel, Eq. (26) can be written as

$$
\exp (\lambda c) \vec{w}=\left(2 \pi \int_{0}^{+\infty} \overrightarrow{\vec{\Phi}} \circ \vec{A} I_{0}(\lambda \boldsymbol{\Delta}) d \boldsymbol{\Delta}\right) \vec{w},
$$

where $I_{0}(\lambda \boldsymbol{\Delta})$ stands for the modified Bessel function of the first kind and order zero [Eq. (4)].

It is easy to see that the matrix inside the brackets in the right-hand side of Eq. (26) becomes, for $n=3$, the matrix $\vec{H}(\lambda)$ given by Eq. (13). We define generally $\vec{H}(\lambda)$ as

$$
\vec{H}(\lambda) \equiv 2 \pi \int_{0}^{+\infty} \overrightarrow{\vec{\Phi}} \circ \overrightarrow{\vec{A}} I_{0}(\lambda \boldsymbol{\Delta}) d \boldsymbol{\Delta} .
$$

Then we can rewrite Eq. (26) as

$$
\exp (\lambda c) \vec{w} \equiv \stackrel{\vec{H}}{H}(\lambda) \vec{w}
$$

and, as mentioned above, the solution for the front speed $c$ is [11]

$$
c=\min _{\lambda>0} \frac{\ln \left[\rho_{1}(\lambda)\right]}{\lambda},
$$

where $\rho_{1}$ is the largest real of the eigenvalues of $\vec{H}(\lambda)$, which is now given by Eq. (28). This new result is the $2 \mathrm{D}$ equivalent to previous results in one dimension [11] for an arbitrary number of stages $n$. However, we stress that $1 \mathrm{D}$ results can be applied only in very special systems (e.g., a population spreading along a coast or a river), but most population invasions on the Earth take place in two dimensions. Therefore, in most cases $1 \mathrm{D}$ results cannot be applied. Indeed, the Bessel function $I_{0}(\lambda \boldsymbol{\Delta})$ appears in Eq. (28), whereas it does not appear at all for populations spreading in one dimension [11]. In Sec. III B we present some numerical 1D and 2D overlapping results for a population with a large number of stages.

\section{APPLICATION}

In previous sections we have developed a model to calculate front speed for a general case of structured populations in two dimensions. As an example, in this section we will use the matrices in Eq. (25) and parameter values appropriate to study a specific tree species, namely, the yellow poplar ( $\mathrm{Li}$ riodendron tulipifera). This species was already considered in Ref. [10], but only by means of a nonoverlapping generation model. Below we apply our overlapping-generations model, and compare to simulations of structured populations.
Furthermore, at the end of this paper we will tackle the question of how important the effect of overlapping generations is, by comparing our results with those of the nonoverlapping-generations model [10].

\section{A. Molecular dynamics simulations}

We are not dealing with a differential but with an integrodifference set of equations in two dimensions [Eq. (25)]. Therefore, numerical simulations in this paper will not be based on finite-step approximations to partial derivatives but on molecular dynamics. We have performed simulations on a 2D grid, with nearest neighbors separated by a distance $\boldsymbol{\Delta}_{0}$. Initially $p(x, y, 0)=1$ at $(x, y)=(0,0)$ and $p(x, y, 0)=0$ elsewhere. At each time step, we compute the new number density of trees $p(x, y, t+1)$ at all nodes of the grid as follows.

First of all, because of the structure of the population, not all of the individuals can produce seeds. We need to distinguish the adult population (i.e., individuals that take a role in a reproduction event) from juvenile individuals (i.e., those that cannot yet produce seeds). In order to do so, we introduce a second field $j(x, y, t)$ that accounts for the juvenile individuals. While the first field accounts for the total population density [i.e., $p(x, y, t)$ includes juveniles and adults], the second one contains only the juvenile population density $j(x, y, t)$. The number of individuals being available to reproduce [namely $a(x, y, t)]$ is simply the difference between $p(x, y, t)$ and $j(x, y, t)$. In each time step (or iteration), our computer program calculates the seed production $R_{0} a(x, y, t)$ at every node and then redistributes this value among all grid nodes by using the dispersal kernel. Moreover, we also take into account when an individual is too old to be fertile (i.e., when it reaches the typical life span $l$ of the species considered). This is done by removing such individuals from the calculations after each time step (i.e., after each year).

We will focus our attention on the effects of the population structure (in order to solve the front speed problem), instead of studying complicated dispersal kernels. Therefore, our simulations apply a simple kernel such that, when a seed is produced, it either remains at the same node where it was generated (with a probability $p_{e}$ ) or it is dispersed to one of the four nearest-neighbor nodes located at distance $\Delta_{0}$ [with probability $\left.\left(1-p_{e}\right) / 4\right]$.

We have performed our 2D simulations by using the typical values measured for the yellow poplar, namely, life span $l=130 \mathrm{yr}$., age at first reproduction $T=20 \mathrm{yr}$., persistence $p_{e}=0.99798$ and characteristic dispersal distance $\boldsymbol{\Delta}_{0}$ $=6000 \mathrm{~m}$ [24]. The results for the front speed are shown in Fig. 1, both along the horizontal and along the diagonal direction of the 2D grid (upper and lower triangles in Fig. 1). We also include the average speeds between both directions (circles in Fig. 1).

\section{B. CSRW}

In order to study an overlapping-generations front using a 2D CSRW, first we need to determine the order of the matrices in Eq. (25). Recall that in our structured-population model, every year of an individual's life is thought of as a different stage. From the life span of the yellow poplar [24], 


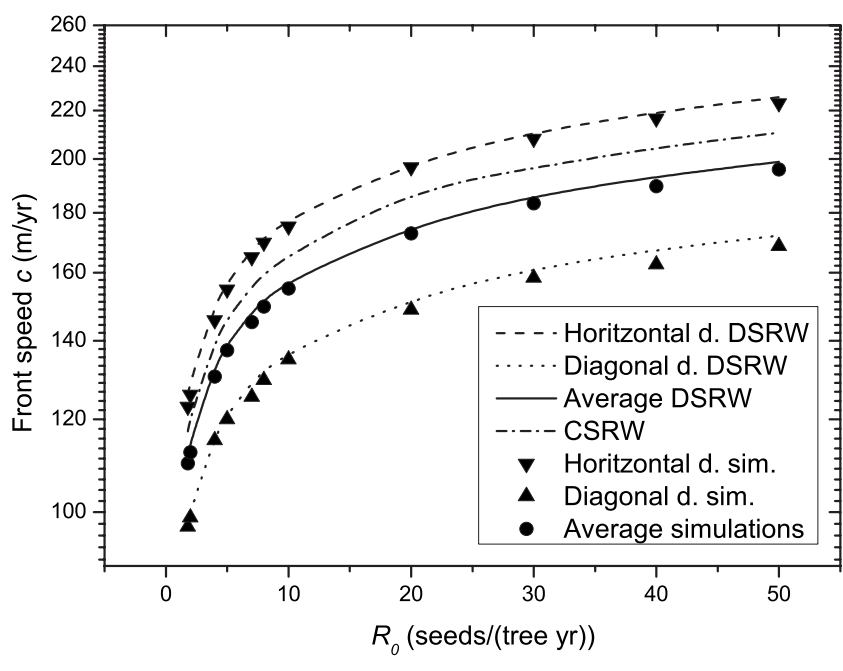

FIG. 1. Front speed in two dimensions versus net reproductive rate. There are relevant differences between the CSRW and the simulations (above 5\%). Because of this, we have also developed a DSRW model that agrees correctly with simulations (differences are always under $3 \%$ ).

namely, 130 years, it follows that Eq. (25) shall be a system of order 130 [25]. Yellow poplar trees begin to produce seeds when they are 20 years old. Therefore, the demographic matrix $\vec{A}$ will be

$$
a_{i j}= \begin{cases}R_{0}, & \text { if } i=1 \text { and } j \geq 20 \\ 1, & \text { if } i=j+1 \\ 0, & \text { otherwise. }\end{cases}
$$

Note that the structure of this matrix is the same as that of Eq. (22), with the difference that the matrix (31) is of order 130. In order to obtain the dispersal kernel matrix $\vec{\Phi}$, we recall that, as explained above, the dispersal kernel per unit length reads

$$
\varphi(\Delta)= \begin{cases}\left(1-p_{e}\right), & \text { if } \boldsymbol{\Delta}=\boldsymbol{\Delta}_{0} \\ p_{e}, & \text { if } \boldsymbol{\Delta}=0 \\ 0, & \text { otherwise }\end{cases}
$$

where $\boldsymbol{\Delta}_{0}$ and $p_{e}$ take the typical values of long-distance dispersal and persistence for the yellow poplar, namely, $p_{e}$ $=0.99798$ and $\boldsymbol{\Delta}_{0}=6000 \mathrm{~m}$ [24]. On the other hand,

$$
\Phi_{i j}= \begin{cases}\frac{\varphi(\boldsymbol{\Delta})}{2 \pi \boldsymbol{\Delta}} & \text { if } i=1 \text { and } j>20 \\ \delta^{2 \mathrm{D}}(\boldsymbol{\Delta}) & \text { otherwise, }\end{cases}
$$

where $\delta^{2 \mathrm{D}}(\boldsymbol{\Delta})$ is the Dirac delta function in two dimensions, namely [see Eq. (5)]

$$
\delta^{2 \mathrm{D}}(\boldsymbol{\Delta})=\frac{\delta^{1 \mathrm{D}}(\boldsymbol{\Delta})}{2 \pi \boldsymbol{\Delta}},
$$

and its normalization condition reads

$$
\int_{0}^{\infty} \int_{0}^{2 \pi} \delta^{2 \mathrm{D}}(\boldsymbol{\Delta}) \boldsymbol{\Delta} d \boldsymbol{\Delta} d \theta=1 .
$$

TABLE II. Front speeds in one and two dimensions (in $\mathrm{m} / \mathrm{yr}$ ) and the error of the $1 \mathrm{D}$ speed (relative to the 2D speed $c$ ) for $n$ $=130$ stages (yellow poplar) and several values of the net fecundity $R_{0}$ [measured in seeds/(tree* $\left.\left.\mathrm{yr}\right)\right]$.

\begin{tabular}{rccc}
\hline \hline$R_{0}$ & $c_{1 \mathrm{D}}$ & $c$ & $\begin{array}{c}\text { Error } \\
(\%)\end{array}$ \\
\hline 4 & 164.1 & 139.8 & 14.8 \\
10 & 193.7 & 165.8 & 14.4 \\
40 & 236.8 & 204.5 & 13.6 \\
\hline \hline
\end{tabular}

We have used these equations into Eqs. (28)-(30) to compute invasion speeds for different values of the net reproductive rate $R_{0}$. The results are shown in Fig. 1. Both the simulations and the CSRW predictions are seen to increase with increasing values of $R_{0}$, as it was to be expected intuitively. However, there are some differences (larger than $5 \%$ ) between the CSRW and the average simulations. This effect is due to the fact that, while the CSRW describes jumps into a ring of radius $\boldsymbol{\Delta}_{0}$, the numerical simulations describe jumps to four discrete points (i.e., the four nearest neighbors of the grid). The consistency between the analytic and numeric results can be improved by using a discrete-space randomwalk model (DSRW). In such an analytical model, seeds and trees can only lie on discrete points of the space (similarly to what happens in the numerical simulations). Recently, a DSRW model for nonstructured (i.e., nonoverlapping) populations of persistence $p_{e}$ has been proposed [20]. In the next section we will extend that nonoverlapping DSRW model to our structured populations, and we will apply it to the yellow poplar.

Before closing this section, a numerical comparison to the 1D overlapping model can be useful. Whereas Sec II B 1 and Table I include such a comparison for three-stage populations, here we present it for a yellow poplar population (because it has a large number of stages). Similarly to Eq. (17), elements $h_{i j}^{1 \mathrm{D}}$ of the corresponding yellow poplar $\overrightarrow{\overline{H_{1 \mathrm{D}}}}(\lambda) \mathrm{ma}-$ trix take the form

$h_{i j}^{1 \mathrm{D}}=\left\{\begin{array}{cl}R_{0}\left[\begin{array}{cl}p_{e}+\frac{1}{2}\left(1-p_{e}\right) \exp \left(-\lambda \Delta_{0}\right) \\ +\frac{1}{2}\left(1-p_{e}\right) \exp \left(\lambda \Delta_{0}\right)\end{array}\right], & \text { if } i=1, j \geq T \\ 1, & \text { if } i=j+1 \\ 0, & \text { elsewhere }\end{array}\right.$

and the front speed is given by Eq. (18). Using the values of the persistence and jump distance above (i.e., $p_{e}=0.99798$ and $\boldsymbol{\Delta}_{0}=6000 \mathrm{~m}$ ) we have calculated the front speed for several values of $R_{0}$. The results are shown in Table II. It 
follows that for a yellow poplar population, the overestimation of the 1D model is still important, but a bit lower than in the three-stage example (Table I). In all cases, the overestimation is always higher than $10 \%$ (and it increases for decreasing values of the net reproductive rate). Thus we remark the convenience of using a 2D model, also in the case of populations with large number of stages.

\section{DSRW}

\section{Horizontal direction $\left(0^{\circ}\right)$}

Assuming nonoverlapping generations, the DSRW model corresponds to the following evolution equation [20]

$$
\begin{aligned}
p(x, y, t+T)= & R_{0}\left\{p_{e} p(x, y, t)+\frac{\left(1-p_{e}\right)}{4}\right. \\
& \times\left[p\left(x \pm \Delta_{0}, y, t\right)\right. \\
& \left.\left.+p\left(x, y \pm \Delta_{0}, t\right)\right]\right\}
\end{aligned}
$$

In contrast, the dynamics of a population with overlapping generations will be driven by the set

$$
\begin{gathered}
p_{1}(x, y, t+1)=R_{0} \sum_{i=T}^{n}\left\{p_{e} p_{i}(x, y, t)+\frac{\left(1-p_{e}\right)}{4}\right. \\
\times\left[p_{i}\left(x \pm \Delta_{0}, y, t\right)\right. \\
\left.\left.+p_{i}\left(x, y \pm \Delta_{0}, t\right)\right]\right\}, \\
p_{2}(x, y, t+1)=p_{1}(x, y, t), \\
p_{3}(x, y, t+1)=p_{2}(x, y, t), \\
\vdots \\
p_{n}(x, y, t+1)=p_{n-1}(x, y, t),
\end{gathered}
$$

where, as in the previous sections, for simplicity we have assumed that all stages have the same reproductive and dispersive behavior, that individuals in stage $i$ will be in stage $i+1$ a year later, which trees cannot reproduce before the so-called generation time $T$, etc.

As usual, we look for constant-shape solutions with the form $p_{i}(x, y, t)=w_{i} \exp [-\lambda(x-c t)]$. We remark that looking for such solutions implies that we will evaluate the speed of the front along the horizontal direction (i.e., the $0^{\circ}$ direction relative to the $x$ axis). In this way, we obtain the following equations:

$$
\begin{gathered}
w_{1} \exp (\lambda c)=R_{0} \sum_{i=T}^{n} w_{i}\left[\frac{p_{e}+1}{2}+\frac{1-p_{e}}{2} \cosh \left(\lambda \boldsymbol{\Delta}_{0}\right)\right], \\
w_{2} \exp (\lambda c)=w_{1}, \\
w_{3} \exp (\lambda c)=w_{2},
\end{gathered}
$$

$$
w_{n} \exp (\lambda c)=w_{n-1} .
$$

Finally, we use matrix notation to rewrite the system in more compact form,

$$
\exp (\lambda c) \vec{w} \equiv \stackrel{\vec{H}}{H}(\lambda) \vec{w}
$$

where it is easy to see that the reaction-dispersal matrix of population takes the form

$$
h_{i j}= \begin{cases}R_{0}\left[\frac{p_{e}+1}{2}+\frac{1-p_{e}}{2} \cosh \left(\lambda \Delta_{0}\right)\right], & \text { if } i=1, j \geq T \\ 1, & \text { if } i=j+1 \\ 0, & \text { elsewhere }\end{cases}
$$

and the front speed will be found by means of Eq. (30).

\section{Diagonal direction $\left(45^{\circ}\right)$}

We now introduce new coordinate axes $\left(x^{\prime}, y^{\prime}\right)$ on the diagonal direction, i.e., $X^{\prime}$ and $Y^{\prime}$ axes rotated $45^{\circ}$ with respect to the $X$ and $Y$ axes. Then, for nonoverlapping generations the population density evolves according to [20]

$$
\begin{aligned}
p\left(x^{\prime}, y^{\prime}, t+1\right)= & R_{0}\left\{p_{e} p\left(x^{\prime}, y^{\prime}, t\right)+\frac{\left(1-p_{e}\right)}{4}\right. \\
& \times\left[p\left(x^{\prime}+\frac{\boldsymbol{\Delta}_{0}}{\sqrt{2}}, y^{\prime} \pm \frac{\boldsymbol{\Delta}_{0}}{\sqrt{2}}, t\right)\right. \\
& \left.\left.+p\left(x^{\prime}-\frac{\boldsymbol{\Delta}_{0}}{\sqrt{2}}, y^{\prime} \pm \frac{\boldsymbol{\Delta}_{0}}{\sqrt{2}}, t\right)\right]\right\} .
\end{aligned}
$$

However, for overlapping generations we have instead

$$
\begin{gathered}
p_{1}\left(x^{\prime}, y^{\prime}, t+1\right)=R_{0} \sum_{i=T}^{n}\left\{p_{e} p_{i}\left(x^{\prime}, y^{\prime}, t\right)+\frac{\left(1-p_{e}\right)}{4}\right. \\
\times\left[p_{i}\left(x^{\prime}+\frac{\boldsymbol{\Delta}_{0}}{\sqrt{2}}, y^{\prime} \pm \frac{\boldsymbol{\Delta}_{0}}{\sqrt{2}}, t\right)\right. \\
\left.\left.+p_{i}\left(x^{\prime}-\frac{\boldsymbol{\Delta}_{0}}{\sqrt{2}}, y^{\prime} \pm \frac{\boldsymbol{\Delta}_{0}}{\sqrt{2}}, t\right)\right],\right\} \\
p_{2}\left(x^{\prime}, y^{\prime}, t+1\right)=p_{1}\left(x^{\prime}, y^{\prime}, t\right), \\
p_{3}\left(x^{\prime}, y^{\prime}, t+1\right)=p_{2}\left(x^{\prime}, y^{\prime}, t\right), \\
\vdots \\
p_{n}\left(x^{\prime}, y^{\prime}, t+1\right)=p_{n-1}\left(x^{\prime}, y^{\prime}, t\right) .
\end{gathered}
$$

Next, we look for constant-shape solutions, now with the form $p_{i}\left(x^{\prime}, y^{\prime}, t\right)=w_{i} \exp \left[-\lambda\left(x^{\prime}-c t\right)\right]$. We obtain from the set (42),

$$
w_{1} \exp (\lambda c)=\sum_{i=T}^{n} w_{i} R_{0}\left[p_{e}+\left(1-p_{e}\right) \cosh \left(\lambda \frac{\Delta_{0}}{\sqrt{2}}\right)\right],
$$




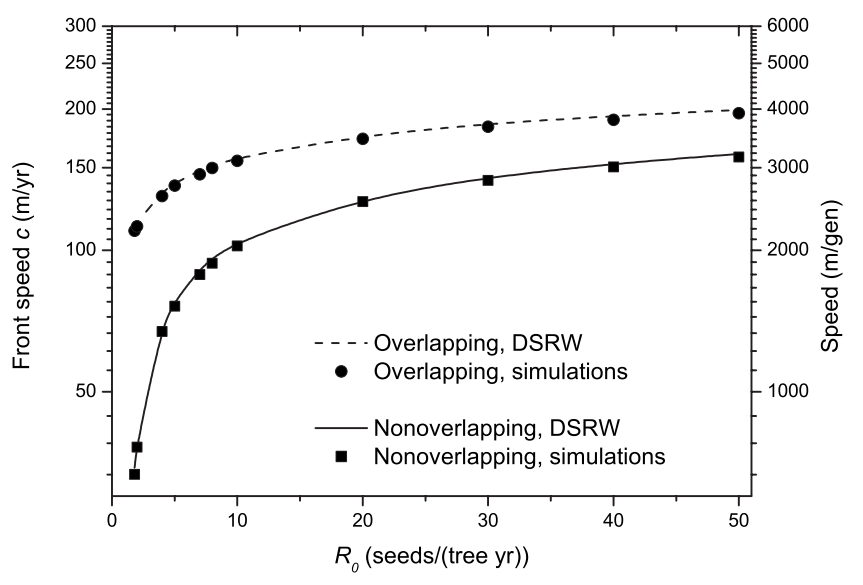

FIG. 2. Front speed in two dimensions versus net reproductive rate. Both nonoverlapping (squares) and overlapping (circles) DSRW results correspond to the average between the horizontal and diagonal spread directions (Sec. III C). Each model agrees perfectly with its corresponding simulations (continuous and dashed lines). The effect of considering overlapping generations becomes more important as lower values of $R_{0}$ are considered.

$$
\begin{gathered}
w_{2} \exp (\lambda c)=w_{1}, \\
w_{3} \exp (\lambda c)=w_{2}, \\
\cdots \\
w_{n} \exp (\lambda c)=w_{n-1},
\end{gathered}
$$

the corresponding reaction-dispersal matrix takes the form

$$
h_{i j}= \begin{cases}\sum_{i=T}^{n} R_{0}\left[p_{e}+\left(1-p_{e}\right) \cosh \left(\lambda \frac{\boldsymbol{\Delta}_{0}}{\sqrt{2}}\right)\right], & \text { if } i=1, j \geq T \\ 1, & \text { if } i=j+1 \\ 0, & \text { elsewhere, }\end{cases}
$$

and the front speed shall be obtained with the usual procedure [Eq. (30)].

In Fig. 1, the DSRW calculations have been performed for both the horizontal and the diagonal direction (dashed and dotted curves, respectively). We can see an almost perfect agreement between DSRW and simulations (differences are always under $2 \%$ after averaging results along both directions). Thus the DSRW is the model that agrees closer to the simulations, even when overlapping generations are considered. This shows the validity of the simulation results in Fig. 1 , as well as the origin of the discrepancies between the CSRW and the simulations in Fig. 1.

\section{NONOVERLAPPING VERSUS OVERLAPPING GENERATIONS MODEL}

The nonoverlapping approximation has an obvious advantage, namely, that computation times are much shorter [26]. Figure 2 shows invasion speeds of yellow poplar fronts cal- culated with both the classical, nonoverlapping model and our overlapping model (both in two dimensions). From Fig. 2 we see that the behavior of both nonoverlapping (squares) and overlapping (circles) front speeds are similar. As it was expected, the overlapping model yields faster invasion speeds. Actually, both models tend to the same asymptotic speed for very high values of the net reproductive rate $\left(R_{0}\right.$ $\rightarrow \infty$ ). This happens because in the limit $R_{0} \rightarrow \infty$, the contribution of the youngest individuals able to produce seeds on the front clearly becomes much more important than that from older trees, and that is precisely the main assumption of the nonoverlapping approximation. In contrast, multiple reproduction events have a very important effect on the front speed for lower, realistic values of the net reproductive rate $R_{0}$. Figure 2 shows substantial differences between the nonoverlapping and the overlapping case for $2<R_{0}<10$, which is within a realistic range for the yellow poplar [24]. The speed predicted by the classical nonoverlapping model is less than $\frac{1}{3}$ of that predicted by our overlapping model.

From Fig. 2, it is also very interesting that the overlapping-generations effect allows the survival and spread of the population even for $R_{0}<1$ (the precise threshold will of course depend on the features of the species considered), whereas the nonoverlapping model predicts extinction if $R_{0}$ $<1$ [21]. This effect also shows that the overlappinggenerations model can be very important to perform realistic predictions on the fate of biological populations. It can also be very useful in more detailed studies involving, e.g., nonsteady or random values of $R_{0}$ (simulating climate change, drought, or epidemic episodes), different productivities $R_{0 i}$ for each stage $i$, etc. In contrast, the nonoverlapping model is not suitable to analyze such situations because it will break down as soon as values $R_{0}<1$ are considered.

Concerning Reid's paradox of rapid tree migration, our previous work suggests that it can be solved by taking into account bimodal dispersal kernels (at least, as far as the order of magnitude of the speed is concerned). The structure of the population, introduced in this paper, makes the overlappinggenerations model more realistic. Therefore, we expect its results to be closer to real measurements of, for example, postglacial recolonization fronts (even though such direct comparisons are not yet feasible for a variety of tree species, because of the uncertainties in the measurements of survival rates and dispersal kernels).

\section{CONCLUSIONS}

In this paper we have presented a model that considers the effect of overlapping generations (or, more generally, the stage-structure) of populations that spread on a twodimensional space. Describing such populations with a matrix notation is useful, and notably simpler, in order to solve the equations that drive the reaction-dispersal dynamics. Actually, in Secs. II B, III B, and III C, writing down the reaction-dispersal matrix $\overrightarrow{\vec{H}}$ is one of the main steps in the resolution of the front speed problem. This reaction-dispersal matrix contains all of the information concerning the population (i.e., parameters such as the persistence, stage-to-stage transitions, characteristic dispersal distance, etc.) necessary 
to apply Eq. (30) to a specific species and, thus, to obtain its front speed. Generally, the construction of two simpler matrices (namely the demographic matrix and the dispersal one) is quite useful to create the reaction-dispersal matrix (as it is done for the CSRW in Sec. III B). However, in some cases it

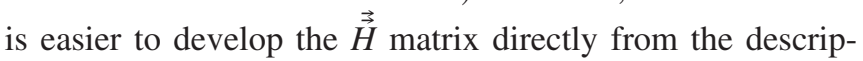
tion of the population. That is the case of the DSRW explained in Sec. III C.

Figure 1 shows remarkable differences between the CSRW analytical results and the corresponding moleculardynamics simulations. The same problem was solved previously in the nonoverlapping case using the DSRW description [20]. Therefore, in Sec. III C we have extended the DSRW to the overlapping case, obtaining an almost perfect agreement with the simulations (see Fig. 1).

We have compared the nonoverlapping and the overlapping models (Fig. 2.) Overlapping-generations fronts are always faster than nonoverlapping ones (except in the limit $R_{0} \rightarrow \infty$ ). The overlapping-generations approximation can seriously underestimate the speed. Indeed, for realistic param- eter values, the speed predicted by the classical, nonoverlapping model is less than $\frac{1}{3}$ of that predicted by our overlapping model. This shows the interest of the problem solved in the present paper. It is also important that the nonoverlapping-generations approximation can wrongly predict extinction for low values of the net reproductive rate $R_{0}$. Our 2D overlapping-generations model can be easily extended to describe more complicated situations that cannot the tackled within the nonoverlapping approximation (e.g., a net reproductive rate $R_{0}$ that decreases with increasing age of individuals), so future works could report a variety of new applications of the results reported in the present paper.

\section{ACKNOWLEDGMENTS}

Funded by European Commission under Grant No. 28192-FEPRE, MICINN under Grant No. FIS-2009-13050, and Generalitat de Catalunya under Grant No. 2009-SGR374.
[1] J. Fort and V. Méndez, Rep. Prog. Phys. 65, 895 (2002).

[2] W. van Saarloos, Phys. Rep. 386, 29 (2003).

[3] J. Fort and T. Pujol, Rep. Progr. Phys. 71, 086001 (2008).

[4] J. Fort and V. Méndez, Phys. Rev. Lett. 89, 178101 (2002).

[5] J. Fort, J. Pérez, E. Ubeda, and F. J. García, Phys. Rev. E 73, 021907 (2006).

[6] N. Vladimirova and R. Rosner, Phys. Rev. E 67, 066305 (2003).

[7] T. Pujol, J. Fort, J. R. González, L. Montoro, and M. Pelegrí, Physica A 387, 1987 (2008).

[8] J. Fort and V. Méndez, Phys. Rev. Lett. 82, 867 (1999).

[9] J. Fort, D. Jana and J. M. Humet, Phys. Rev. E 70, 031913 (2004).

[10] J. Fort, J. Appl. Phys. 101, 094701 (2007).

[11] M. Neubert and H. Caswell, Ecology 81, 1613 (2000).

[12] H. Caswell, Matrix Population Models (Sinauer, Sunderland, 1989)

[13] F. van den Bosch, J. A. J. Metz, and O. Diekmann, J. Math. Biol. 28, 529 (1990).

[14] D. Mollison, Math. Biosci. 107, 255 (1991).

[15] R. Lui, SIAM J. Math. Anal. 13, 913 (1982).

[16] R. Lui, SIAM J. Math. Anal. 13, 938 (1982).

[17] Y. Hosono, Forma 10, 235 (1995).

[18] Y. Hosono, Bull. Math. Biol. 60, 435 (1998).

[19] J. S. Clark, Am. Nat. 152, 204 (1998).

[20] J. Fort, J. Pérez-Losada, and N. Isern, Phys. Rev. E 76, 031913 (2007).

[21] H. F. Weinberger, in Nonlinear Partial Differential Equations and Applications, edited by J. Chadam (Springer, Berlin, 1978).

[22] R. A. Horn and C. R. Johnson, Matrix Analysis (Cambridge University Press, Cambridge, 1985).

[23] H. Caswell, R. Lensink, and M. Neubert, Ecology 84, 1968 (2003).

[24] According to field observations in sites close to those where the dispersal kernel was measured, we estimate the net repro- ductive rate $R_{0}$ of the yellow poplar (Liriodendron Tulipifera) to be in the range of 2-50 seeds/(tree yr.). The age at first reproduction (generation time in the nonstructured model) of the same species is $T=20 \mathrm{yr}$. As shown in [10], the longdistance dispersal component of the kernel has a much more important effect on the front speed than the short-distance component (even if long-distance dispersal happens seldom). Therefore, we use for the distance between cells that from the long-distance component, $\Delta_{0}=6000 \mathrm{~m}$, both in the CSRW and in the DSRW models.

[25] All of the results in this paper have been calculated with square matrices $\overrightarrow{\vec{A}}$ and $\vec{\Phi}$ of order 130 . However, we have observed that in many cases we can obtain accurate results using matrices of lower order. That can be very useful in order to reduce computation times. For instance, omitting all of the elements $a_{i j}$ with $i>65$ and/or $j>65$, we obtain a demographic matrix of order 65. If we do the same for the dispersal kernel matrix and we solve the equations of our overlappinggenerations model, we obtain the same invasion speeds (differences are under the $1 \%$ ) for values of $R_{0}>5$. The reason is that very old individuals do not appear near the leading edge of the invasion front, so for high values of the fecundity $R_{0}$ the front dynamics is driven by younger individuals and the contribution of older ones is not relevant. However we do not give a general description of such an approximation because it depends on the dispersal kernel and demographic characteristics of each species.

[26] Calculation of each overlapping-DSRW speed in Fig. 2 takes about 50 to 80 minutes. We used an Intel Core 2 CPU, T7400 $(2,16 \mathrm{GHz}$ and $2 \mathrm{~GB} \mathrm{RAM})$. We remark that calculation times become much longer as the order of the matrix $\overrightarrow{\vec{H}}$ increases. In contrast, each overlapping-model simulation takes about 10 minutes using the same computer. For nonoverlapping models, calculation times are about ten times shorter than for overlapping models. 